\title{
Stage II Cervical Cancer AJCC v7
}

National Cancer Institute

\section{Source}

National Cancer Institute. Stage II Cervical Cancer A/CC v7. NCI Thesaurus. Code C89551.

Stage II includes: II (T2, N0, M0); IIA (T2a, N0, M0); IIA1 (T2a1, N0, M0); IIA2 (T2a2, N0, M0); IIB (T2b, N0, M0); T2: Cervical carcinoma invades beyond uterus but not to pelvic wall or to lower third of vagina. T2a: Tumor without parametrial invasion. T2a1: Clinically visible lesion $4.0 \mathrm{~cm}$ or less in greatest dimension. T2a2: Clinically visible lesion more than $4.0 \mathrm{~cm}$ in greatest dimension. T2b: Tumor with parametrial invasion. N0: No regional lymph node metastasis. M0: No distant metastasis. (AJCC 7th ed.) 